\title{
Therapeutic Efficacy and Safety of Paeoniae Radix Rubra Formulae in Relieving Hyperbilirubinemia Induced by Viral Hepatitis: A Meta-Analysis
}

\section{OPEN ACCESS}

Edited by:

Adolfo Andrade-Cetto, Universidad Nacional Autónoma de

México, Mexico

Reviewed by:

Jianfei Jiang,

University of Pittsburgh, USA

Fang-Rong Chang,

Kaohsiung Medical University, Taiwan

*Correspondence: Yan-Ling Zhao zhaoyl2855@126.com;

Xiao-He Xiao

xiaoxiaohe302@163.com

${ }^{\dagger}$ These authors have contributed equally to this work

Specialty section:

This article was submitted to

Ethnopharmacology,

a section of the journal

Frontiers in Pharmacology

Received: 07 November 2015 Accepted: 04 March 2016 Published: 22 March 2016

Citation:

Huang $Y-Q$, Ma $X$, Wang J, Zhao $Y-L$, Wang J-B, Chen Z, Zhu Y, Shan L-M, Wei S-Z, Wang J and Xiao X-H (2016)

Therapeutic Efficacy and Safety of

Paeoniae Radix Rubra Formulae in Relieving Hyperbilirubinemia Induced by Viral Hepatitis: A Meta-Analysis. Front. Pharmacol. 7:63. doi: 10.3389/fphar.2016.00063
Yin-Qiu Huang ${ }^{1,2+}$, Xiao Ma ${ }^{1,2 \dagger}$, Jian Wang ${ }^{1+}$, Yan-Ling Zhao ${ }^{2 *}$, Jia-Bo Wang ${ }^{3}$, Zhe Chen ${ }^{1,2}$, Yun Zhu ${ }^{4}$, Li-Mei Shan ${ }^{4}$, Shi-Zhang Wei ${ }^{1,2}$, Ji Wang ${ }^{5}$ and Xiao-He Xiao ${ }^{3 *}$

${ }^{1}$ Pharmacy College, Chengdu University of Traditional Chinese Medicine, Chengdu, China, ${ }^{2}$ Department of Pharmacy, 302 Military Hospital of China, Beijing, China, ${ }^{3}$ China Military Institute of Chinese Medicine, 302 Hospital of People's Liberation Army, Beijing, China, ${ }^{4}$ Department of Integrative Medical Center, 302 Hospital of People's Liberation Army, Beijing, China, ${ }^{5}$ Department of Evidence-Based Medicine and Clinical Epidemiology, West China Hospital, Sichuan University, Chengdu, China

Objective: Hyperbilirubinemia is one of the most devastating pathologies induced by various liver diseases. Formulae related to Paeoniae Radix Rubra (PRR) at high doses have been applied to treat hyperbilirubinemia in traditional Chinese medicine (TCM). The aim of this systematic review and meta-analysis is to assess the efficacy and safety of formulae relevant to high-dose PRR in patients suffering from hyperbilirubinemia induced by viral hepatitis.

Methods: We performed a meta-analysis of randomized-controlled clinical trials to evaluate the efficacy and safety of formulae that apply a high dose of PRR for hyperbilirubinemia. Seven databases were searched until April, 2015. All studies were included according to detailed criteria and assessed for methodological quality. The outcome measurements were recorded for further analysis using the RevMan 5.2.11 software.

Results: Fifteen articles involving 1323 patients with hyperbilirubinemia were included. Formulae with high-dose PRR might promote the efficacy of either a combined application ([OR: 3.98, 95\% Cl $(2.91,5.43)]$; $P<0.01)$ or a single application ([OR: $4.00,95 \% \mathrm{Cl}(1.50,10.68)] ; P<0.01)$ for hyperbilirubinemia. The indices of TBIL, ALT, and AST significantly decreased ([MD: $-75.57,95 \% \mathrm{Cl}(-94.88,-56.26)]$, [MD: -26.54 , 95\% Cl (-36.19, -16.88)], and ([MD: $-28.94,95 \% \mathrm{Cl}(-46.26,-11.61)] ; P<0.01)$, respectively. In addition, formulae with high-dose PRR could enhance the treatment efficacy of hyperbilirubinemia triggered by hepatitis B ([OR: 2.98, 95\% Cl $(1.75,5.05)$ ]; $P<0.01)$. Furthermore, the efficacy was enhanced with an increasing dosage of PRR. Two articles reported that no side effects occurred in clinical trials, and three studies noted that patients presented light digestive tract symptoms.

Conclusion: Formulae relevant to high-dose PRR ameliorate hyperbilirubinemia and might constitute a promising therapeutic approach. For widespread acceptance by practitioners, more rigorously designed multicenter, double-blind, randomized, and large-scale controlled trials are required.

Keywords: Paeoniae Radix Rubra formulae, efficacy and safety, meta-analysis, hyperbilirubinemia, viral hepatitis, high dose Paeoniae Radix Rubra 


\section{INTRODUCTION}

Hyperbilirubinemia presenting high serum bilirubin or severe jaundice is one of the most devastating pathologies in patients with various liver diseases (Duan et al., 2006). Continuous hyperbilirubinemia without proper treatment commonly leads to hepatocyte edema, necrosis, inflammation, and even liver cirrhosis, which can directly threaten quality of life (Garg et al., 2011). Current therapies for hyperbilirubinemia mainly focus on a combination of decreasing serum bilirubin, liver protection, regulating transaminase levels, and nutrition support. However, there are unstable curative rates as well as side effects in the clinic. Ursodeoxycholic acid (UDCA) and Ademetionine are the firstline drugs used to decrease serum bilirubin. The inconsistent efficiency and expensive medical costs are common obstacles for some patients (Hou et al., 2010). Potassium magnesium aspartate as an adjuvant is limited by its efficacy in decreasing serum bilirubin. In addition, glucocorticosteroid therapy, due to side effects and the possibility of symptom rebound, is controversial, particularly when treating hyperbilirubinemia for a long duration (Zhang and Shen, 2011).

Growing evidence indicates that traditional Chinese medicine (TCM), as a complementary and alternative therapy, has an important role in hyperbilirubinemia or jaundice treatment ( $\mathrm{Li}$ et al., 2008). The decoctions of Yin Chen Hao, Yin Chen Si Ni, Da Huang Xiao Shi, Zhi Zi Bai Pi, and Chi Dan Tui Huang have been proven to effectively decrease serum bilirubin and protect hepatocytes (He et al., 2003; Zhou, 2010). In particular, several formulae that reverse blood stasis are commonly applied in hyperbilirubinemia. Paeoniae Radix Rubra (PRR, Chishao in Chinese), the dried root of Paeonia lactiflora Pall, has been used for thousands of years in TCM to treat blood stasis. A series of PRR-relevant formulae, due to their significant function as blood invigorators, have been used as treatments for jaundice for decades and have obtained satisfactory efficacy (Ding et al., 2003). Currently, these formulae have been reported as promising therapies in the clinic and are widely used in China. However, a systematic review on formulae relevant to high-dose PRR for treating hyperbilirubinemia has not been reported. Therefore, this meta-analysis of randomized controlled trials (RCTs) was conducted to assess the clinical value of formulae relevant to high-dose PRR in treating hyperbilirubinemia and to provide a possible complementary and alternative therapy for widespread use by practitioners (Figure 1).

\section{METHODS}

\section{Search Strategy}

Comprehensive searches were performed in seven databases by two researchers. The databases included PubMed, Embase,

Abbreviations: PRR, Paeoniae Radix Rubra; ALT, alanine aminotransferase; AST, aspartate aminotransferase; TBIL, total bilirubin; UDCA, ursodeoxycholic acid; TCM, traditional Chinese medicine; RCTs, randomized control trials; VMIS, VIP medicine information system; CBM, Chinese Biomedical Database; CNKI, China National Knowledge Infrastructure; VHPTP, Viral Hepatitis Prevention and Treatment Programs; $\gamma$-GT, $\gamma$-glutamyl transferase; DBIL, direct bilirubin; PT, prothrombin time; $\mathrm{MD}$, mean difference; $\mathrm{OR}$, odds ratio.
Cochrane Library, Chinese Biomedical Database (CBM), Wanfang, VIP medicine information system (VMIS), and China National Knowledge Infrastructure (CNKI) from inception to April, 2015. Search terms included "TCM" or "PRR" or "Chishao" "Paeonia lactiflora pall" or "Paeonia veitchii Lynch", and "severe jaundice," or "hyperbilirubinemia," and "viral hepatitis" according to databases in the Chinese and English languages. The first author, year of publication, title, and journal name from searched articles were recorded.

\section{Exclusion Criteria}

Studies with the following criteria were excluded: (1) reviews, non-clinical studies, and case observations, (2) the absence of Randomized controlled trials (RCTs), or (3) interventions with another TCM formulae (not including extracted agents) or acupuncture therapies in the control group.

\section{Inclusion Criteria}

According to the advice of a liver specialist, the inclusion criteria were designed as follows: (1) RCTs with patients diagnosed as having hyperbilirubinemia by meeting the criteria of Viral Hepatitis Prevention and Treatment Programs (VHPTP) version 2010, 2005, 2000, 1995, or 1990. (2) Studies must have claimed that patients developed hyperbilirubinemia due to viral hepatitis. In addition, participant serum TBIL was above $171 \mu \mathrm{mol} / \mathrm{L}$ before treatment. (3) PRR served as the main component in the formulae and the high dosage was defined as $>25 \mathrm{~g}$ (two times higher than the prescribed maximum dose of Chinese Pharmacopeia 2010 version). (4) High-dose PRRrelevant formulae alone or as a combined application served as the trial group. Placebo or conventional therapy served as the control group. (5) Outcome measurements must have included one or more of the following indices: total effective rate, alanine aminotransferase (ALT), aspartate aminotransferase (AST), total bilirubin (TBIL), direct bilirubin (DBIL), $\gamma$-glutamyl transferase $(\gamma$-GT), albumin (ALB), and prothrombin time (PT). (6) The total efficacy was coincident with the Guidance for Clinical Research on New Drugs of TCM, i.e., recovery as evidenced by the absence of symptoms and TBIL decreasing to nearly normal levels. Effectiveness was identified by ameliorated symptoms and TBIL decreasing below half of the original value $(85.5 \mu \mathrm{mol} / \mathrm{L})$. Invalidation was defined as symptoms and TBIL remaining unchanged or worsening.

\section{Data Extraction and Risk of Bias Assessment}

Data extraction and quality assessment were independently performed by two researchers, and disagreements were resolved by consensus. Detailed data such as study design, participants' information, interventions, outcome measures, and adverse events were extracted to a conclusive table.

The Cochrane risk of bias tool was used to access the methodological quality of the included RCTs. There were six domains, including random sequence generation (selection bias), allocation concealment (selection bias), blinding of participants and personnel (performance bias), blinding of outcome data 


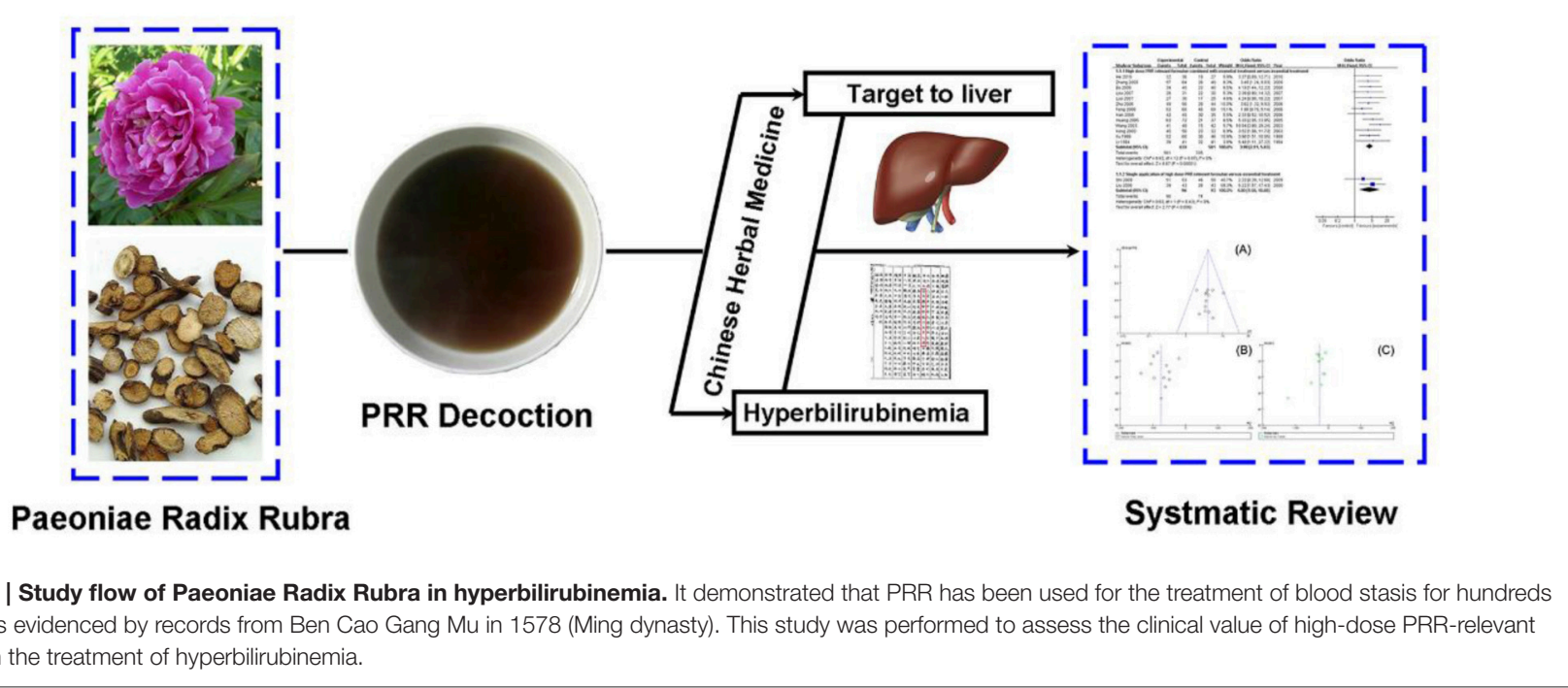

(attrition bias), incomplete outcome data (attrition bias), and selective reporting (reporting bias).

\section{Data Analysis}

Statistical analysis was performed using Cochrane RevMan 5.2.11 (Cochrane Collaboration). Dichotomous data were presented as the odds ratio (OR) and continuous variables as the mean difference (MD) with 95\% confidence intervals (95\% CI). Statistical heterogeneity was assessed by Cochrane's Q-test. Data with low heterogeneity $\left(P \geq 0.10\right.$ and $\left.I^{2} \leq 50 \%\right)$ were analyzed by a fixed-effects model, whereas a random-effect model was used for data with high heterogeneity. A funnel plot was used to assess potential publication bias.

\section{RESULTS}

\section{Characteristics of the Included Studies}

A total of 4300 publications from 7 databases were identified for the initial screen. We removed 1278 duplicated citations, and 2780 were excluded due to irrelevance. The full texts of 242 articles were retrieved for detailed assessment. Of the remaining articles, there were 57 non-randomized controlled studies, 6 animal studies, 97 inconsistent criteria, 40 ineligible PRR doses in the formulae or no mention of dosage, 14 with no PRR in the formulae, 8 summaries, and 5 review articles. Fifteen studies were ultimately used for further meta-analysis (Figure 2).

Fifteen articles involving 1323 subjects with hyperbilirubinemia (729 cases in the trial group, 594 cases in the control group) were included in this study. Because most of the formulae-form therapy was applied in TCM, the 15 studies that had been screened were all published in Chinese. There was no significance in terms of age and sex between these two groups (Table 1). Formulae with high-dose PRR with essential treatment or as a single application were used in the trial group, whereas essential treatment was applied in the control group in all studies. The dose of PRR in the formulae ranged from 30 to $200 \mathrm{~g}$. The treatment duration described in the articles ranged from 2 weeks to 60 days, and 5 articles ( $\mathrm{Li}$ and $\mathrm{Gu}, 1994 ; \mathrm{Xu}$ et al., 1999; Wang et al., 2003; Lou et al., 2007; Bo, 2008) reported adverse events and side effects. No trial reported a follow-up (Table 2).

\section{Methodological Quality of Included Trials}

The methodological quality of the 15 included trials was generally low. According to the Cochrane risk of bias estimation, a randomized allocation of participants was mentioned in all trials. However, only 2 trials (Huang and Yang, 2005; Zhang, 2009) mentioned an appropriate randomized method. Allocation concealment, blinding of participants and blinding of outcome assessment were not recorded in the 15 studies. Six of the 15 articles (Feng et al., 2006; Lou et al., 2007; Luo, 2007; Shi et al., 2009; Zhang, 2009; He, 2010) were at low risk of incomplete outcome data or essential data missing. Selective reporting was generally unclear. Only four articles ( $\mathrm{Li}$ and $\mathrm{Gu}, 1994$; Wang et al., 2003; Luo, 2007; Bo, 2008) reported research protocols, and the result of the detailed indices indicated a low risk of selective reporting. (Figure 3).

\section{Outcome Measures with Subgroup Analysis \\ Efficacy Rate of High-Dose PRR-Relevant Formulae Combined with Essential Treatment or Single Application vs. Essential Treatment}

Thirteen of the 15 articles described the application of formulae on high-dose PRR combined with essential treatment as the trial group. The remaining 2 RCTs (Liu, 2000; Shi et al., 2009) reported a single application of high-dose PRR formulae in the trial group. No heterogeneity was found in both analyses and a fixed effects model was used $(P>0.10)$. The results displayed as OR with $95 \% \mathrm{CI}$ in the combination treatment and single application were [OR: 3.98, 95\% CI $(2.91,5.43)]$ and [OR: 4.00, $95 \%$ CI $(1.50,10.68)]$, respectively. This revealed that formulae of high-dose PRR might promote the treatment efficacy for hyperbilirubinemia $(P<0.01$; Figure 4$)$. 
TABLE 1 | Characteristics of included studies.

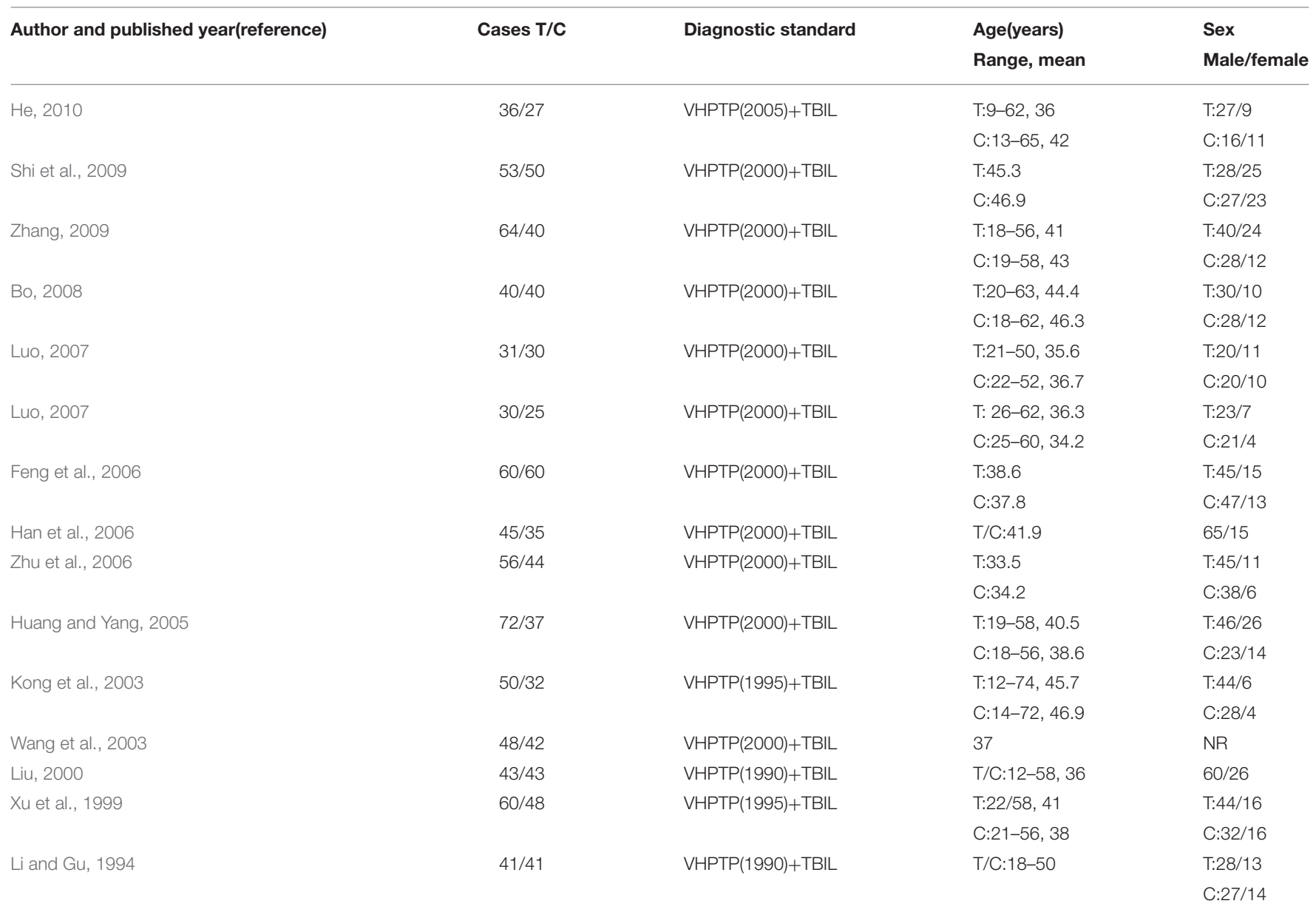

T, trial group; C, control group. NR, no report.

\section{Hyperbilirubinemia Indices of High-Dose PRR-Relevant Formulae vs. Essential Treatment}

The serum TBIL level, which is the direct and crucial index of hyperbilirubinemia, was described in 12 trials. In addition, 3 studies (Huang and Yang, 2005; Lou et al., 2007; Zhang, 2009) reported the serum DBIL level. The serum ALT level was measured in 11 articles, and five studies (Huang and Yang, 2005; Feng et al., 2006; Lou et al., 2007; Shi et al., 2009; Zhang, 2009) recorded the AST level. Serum ALB and $\gamma$-GT were reported in 4 (Kong et al., 2003; Wang et al., 2003; Luo, 2007; Bo, 2008) and two trials (Feng et al., 2006; Shi et al., 2009), respectively. Two trials (Kong et al., 2003; Wang et al., 2003) recorded the PT level. There was heterogeneity in the indices of TBIL, DBIL, ALT, AST, PT, and ALB $(P<0.10)$. Therefore, a random effects model was used for analysis. There was no heterogeneity $(P>0.10)$ in the index of $\gamma$-GT; the fixed-effect model was thus used. The MD with $95 \% \mathrm{CI}$ of serum TBIL, ALT, and AST levels were [MD: $-75.57,95 \%$ CI (-94.88, -56.26)], [MD: -26.54, 95\% CI $(-36.19,-16.88)]$, and [MD: $-28.94,95 \% \mathrm{CI}(-46.26,-11.61)]$, respectively, indicating a significant decrease in the pathologic index in the trial group compared with the control group $(P<0.01)$. The indices of serum DBIL, $\gamma$-GT, and PT were [MD: $-28.41,95 \%$ CI ( -57.81 ,
0.99)], [MD: $-0.03,95 \% \mathrm{CI}(-12.95,12.89)]$, and [MD: -1.29 , $95 \%$ CI $(-3.33,0.74)]$, respectively. There were no significant decreases in the trial group compared with the control group $(P>0.05)$. There was no difference between the trial group and control group for the serum index [MD: ALB 1.84, 95\% CI $(-0.14,3.82)](P>0.05$; Figures 5-7).

\section{Efficacy Rate of High-Dose PRR-Relevant Formulae Combined with Essential Treatment vs. Essential Treatment in Hyperbilirubinemia Patients with Hepatitis B}

Hepatitis B virus is the most common and important cause of hyperbilirubinemia. In this study, the efficacy rate of formulae of high-dose PRR combined with essential treatment was specifically analyzed. There were five articles (Feng et al., 2006; Lou et al., 2007; Luo, 2007; Zhang, 2009; He, 2010) that reported hyperbilirubinemia patients with hepatitis B virus. Based on the fixed effects model $(P>0.10)$, the OR with $95 \% \mathrm{CI}$ was [OR: $2.98,95 \%$ CI $(1.75,5.05)]$. The efficacy rate showed a significant increase in the trial group compared with the control group (Figure 8). 
TABLE 2 | Intervention and outcome measures of included studies.

\begin{tabular}{|c|c|c|c|c|c|}
\hline \multirow[t]{2}{*}{ Study ID } & \multicolumn{2}{|c|}{ Intervention } & \multirow{2}{*}{$\begin{array}{l}\text { Duration/follow } \\
\text { up }\end{array}$} & \multirow[t]{2}{*}{ Adverse events } & \multirow[t]{2}{*}{ Outcome measures } \\
\hline & $\begin{array}{l}\text { Trials group (high-dose } \\
\text { PRR-relevant formulae) }\end{array}$ & $\begin{array}{l}\text { Control group (essential } \\
\text { treatment) }\end{array}$ & & & \\
\hline $\mathrm{He}, 2010$ & $\begin{array}{l}\text { Tui Huang Decoction (PRR } \\
60 \mathrm{~g})+ \text { Essential treatment }\end{array}$ & $\begin{array}{l}\text { Diammonium glycyrrhizinate } \\
+ \text { Vitamin+Inosine+Ku } \\
\text { Huang injection potassium } \\
\text { magnesium } \\
\text { aspartate+Reduced } \\
\text { glutatione }\end{array}$ & 6 weeks/NR & NR & $\begin{array}{l}\text { Total efficacy rate, ALT, } \\
\text { TBIL }\end{array}$ \\
\hline Shi et al., 2009 & $\begin{array}{l}\text { Qing Re Liang Xue Jie Du } \\
\text { Decoction (PRR } 30 \mathrm{~g} \text { ) }\end{array}$ & $\begin{array}{l}\text { Hepatocyte growth } \\
\text { promotion +Liver } \\
\text { protection+Improving } \\
\text { minicirculation }\end{array}$ & 2 weeks/NR & NR & $\begin{array}{l}\text { Total efficacy rate, ALT, } \\
\text { AST, TBIL, } \gamma \text {-GT, PT }\end{array}$ \\
\hline Zhang, 2009 & $\begin{array}{l}\text { Tui Huang Decoction (PRR } \\
30 \mathrm{~g})+ \text { Essential treatment }\end{array}$ & $\begin{array}{l}\text { Diammonium glycyrrhizinate } \\
\text { +Inosine+Vitamin+Reduced } \\
\text { glutatione+glucuronolactone }\end{array}$ & 30 days/NR & NR & $\begin{array}{l}\text { Total efficacy rate, ALT, } \\
\text { AST, TBIL, DBIL }\end{array}$ \\
\hline Bo, 2008 & $\begin{array}{l}\text { Jia Wei Wen Dan } \\
\text { Decoction (PRR } 30 \\
\text { g)+Essential treatment }\end{array}$ & $\begin{array}{l}\text { Diammonium glycyrrhizinate } \\
\text { +Potassium magnesium } \\
\text { aspartate+Vitamin }\end{array}$ & 30 days/NR & $\mathrm{NO}$ & $\begin{array}{l}\text { Total efficacy rate, ALT, } \\
\text { ALB, TBIL }\end{array}$ \\
\hline Luo, 2007 & $\begin{array}{l}\text { Tui Huang Decoction (PRR } \\
30 \mathrm{~g})+ \text { Essential treatment }\end{array}$ & $\begin{array}{l}\text { Diammonium glycyrrhizinate } \\
\text { +Potassium magnesium } \\
\text { aspartate+Vitamin }\end{array}$ & 6 weeks/NR & T: 1 case diarrhea C: NO & $\begin{array}{l}\text { Total efficacy rate, ALT, } \\
\text { AST, TBIL, DBIL }\end{array}$ \\
\hline Luo, 2007 & $\begin{array}{l}\text { Jie Du Huo Xue Decoction } \\
\text { (PRR } 60 \mathrm{~g})+ \text { Essential } \\
\text { treatment }\end{array}$ & $\begin{array}{l}\text { Diammonium glycyrrhizinate } \\
\text { +Potassium magnesium } \\
\text { aspartate +Reduced } \\
\text { glutatione }\end{array}$ & 30 days/NR & NR & $\begin{array}{l}\text { Total efficacy rate, ALT, } \\
\text { TBIL, ALB }\end{array}$ \\
\hline Feng et al., 2006 & $\begin{array}{l}\text { Da Huang Chi Shao } \\
\text { Decoction (PRR 30-60 } \\
\text { g)+Essential treatment }\end{array}$ & $\begin{array}{l}\text { Liver Protection+symptom } \\
\text { Treatment }\end{array}$ & 60 days/NR & NR & $\begin{array}{l}\text { Total efficacy rate, ALT, } \\
\text { AST, TBIL, } \gamma \text {-GT }\end{array}$ \\
\hline Han et al., 2006 & $\begin{array}{l}\text { Chi Ze Decoction (PRR } \\
100 \mathrm{~g})+ \text { Essential } \\
\text { treatment }\end{array}$ & $\begin{array}{l}\text { Liver Protection+symptom } \\
\text { Treatment }\end{array}$ & 1 month/NR & NR & $\begin{array}{l}\text { Total efficacy rate, ALT, } \\
\text { TBIL }\end{array}$ \\
\hline Zhu et al., 2006 & $\begin{array}{l}\text { PRR } 200 \mathrm{~g}+\text { Essential } \\
\text { treatment }\end{array}$ & Dan Shen injection+Vitamin & 1 month/NR & NR & Total efficacy rate \\
\hline Huang and Yang, 2005 & $\begin{array}{l}\text { Liang Xue Hua Yu } \\
\text { Decoction(PRR } 50 \\
\text { g)+Essential treatment }\end{array}$ & $\begin{array}{l}\text { Vitamin+Hepatocyte } \\
\text { growth-promoting } \\
\text { factors+Potassium } \\
\text { magnesium aspartate+ALB }\end{array}$ & 30 days/NR & NR & $\begin{array}{l}\text { Total efficacy rate, ALT, } \\
\text { AST, TBIL, DBIL }\end{array}$ \\
\hline Kong et al., 2003 & $\begin{array}{l}\text { PRR Relevant } \\
\text { Formulae(PRR 30-60 } \\
\text { g)+Essential treatment }\end{array}$ & $\begin{array}{l}\text { Diammonium glycyrrhizinate } \\
\text { +Potassium magnesium } \\
\text { aspartate+Vitamin+Hepatocyte } \\
\text { growth-promoting factors }\end{array}$ & 6 weeks/NR & NR & $\begin{array}{l}\text { Total efficacy rate, ALT, } \\
\text { TBIL, ALB, PT }\end{array}$ \\
\hline Wang et al., 2003 & $\begin{array}{l}\text { Tui Gao Huang } \\
\text { Decoction(PRR } 120 \\
\text { g)+Essential treatment }\end{array}$ & $\begin{array}{l}\text { Vitamin+Adenosine } \\
\text { disodiu+Yin Zhi Huang } \\
\text { injection }\end{array}$ & 4 weeks/NR & $\begin{array}{l}\text { T: Few with light digestive } \\
\text { tract side effect C: NO }\end{array}$ & $\begin{array}{l}\text { Total efficacy rate, ALT, } \\
\text { TBIL, ALB, PT }\end{array}$ \\
\hline Liu, 2000 & $\begin{array}{l}\text { PRR relevant } \\
\text { formulae(PRR 100-150 g) }\end{array}$ & $\begin{array}{l}\text { Potassium magnesium } \\
\text { aspartate } \\
\text { +Vitamin+Bifendate }\end{array}$ & 2 weeks/NR & NR & Total efficacy rate \\
\hline Xu et al., 1999 & $\begin{array}{l}\text { Chi Zhi Huang } \\
\text { Decoction(PRR } 90 \\
\text { g)+Essential treatment }\end{array}$ & $\begin{array}{l}\text { Potassium magnesium } \\
\text { aspartate } \\
\text { +Vitamin+Adenosine } \\
\text { Disodiu+Glucuronolactone+ } \\
\text { Silymarin }\end{array}$ & 30 days/NR & $\begin{array}{l}\text { T: Few with light digestive } \\
\text { tract side effect C: NO }\end{array}$ & Total efficacy rate \\
\hline Li and Gu, 1994 & $\begin{array}{l}\text { PRR relevant } \\
\text { formulae(PRR 60-120 } \\
\text { g)+Essential treatment }\end{array}$ & $\begin{array}{l}\text { Silymarin+Vitamin+Inosine+ } \\
\text { Potassium magnesium } \\
\text { aspartate+Yin Zhi Huang } \\
\text { injection }\end{array}$ & 30 days/NR & $\mathrm{NO}$ & Total efficacy rate, TBIL \\
\hline
\end{tabular}

T, trial group, C, control group. NR, no report. 


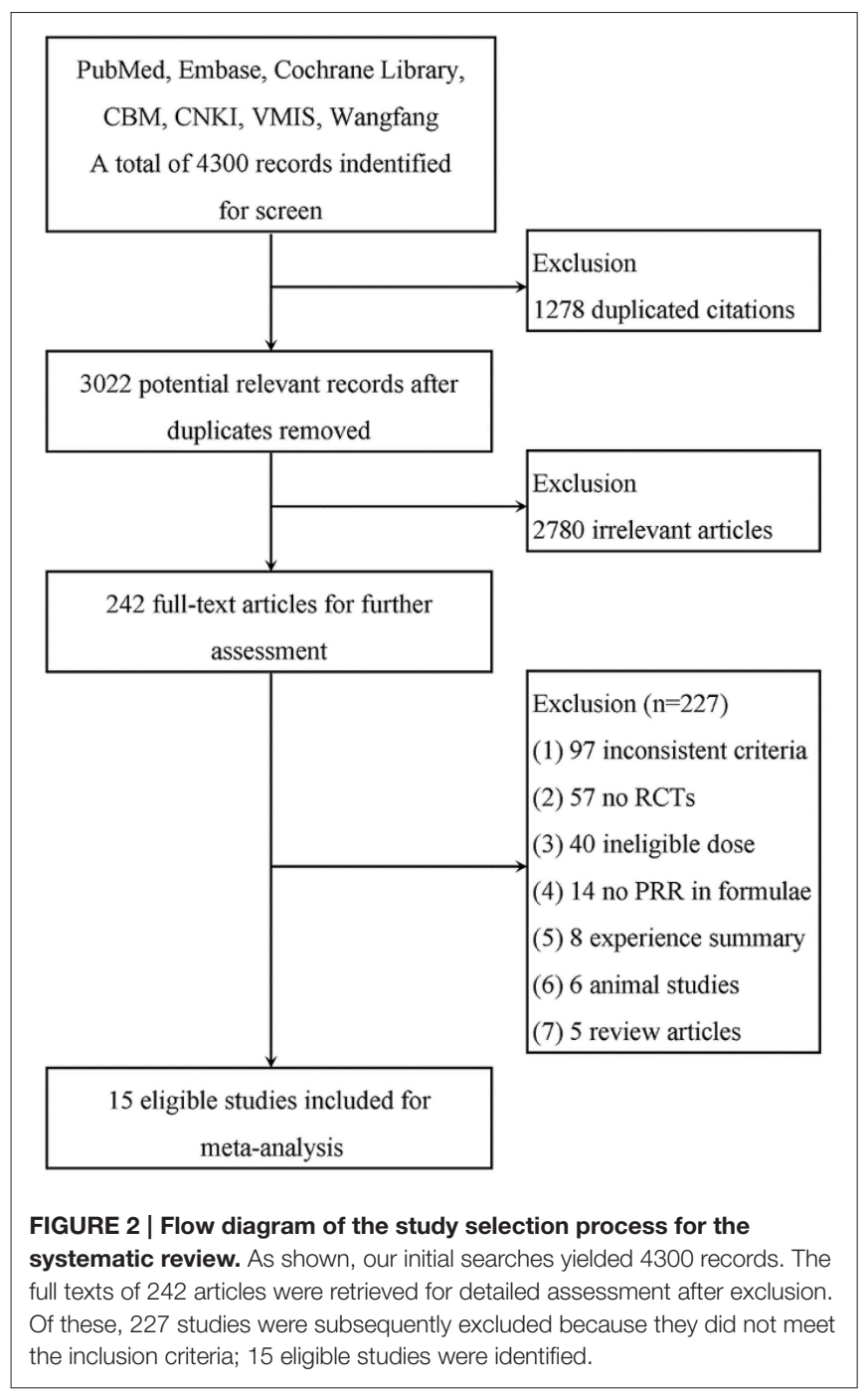

\section{Efficacy Rate of Different Ranges of High-Dose PRR-Relevant Formulae Combined with Essential Treatment vs. Essential Treatment in Hyperbilirubinemia}

Because there was a wide range of high-dose PRR from 30 to 200 $\mathrm{g}$, a subgroup analysis of different ranges of formulae of high-dose PRR was needed. We classified these studies into three subgroups (PRR in 30-60 g, PRR in 60-120 g, and PRR greater than $120 \mathrm{~g}$ ) according to the dose application. There were six studies (Kong et al., 2003; Huang and Yang, 2005; Feng et al., 2006; Lou et al., 2007; Bo, 2008; Zhang, 2009) ascribed to the 30-60 g subgroup and five studies ( $\mathrm{Li}$ and Gu, 1994; Xu et al., 1999; Han et al., 2006; Luo, 2007; He, 2010) ascribed to the 60-120 g subgroup. In addition, the remaining 2 studies (Wang et al., 2003; Zhu et al., 2006) were classified to the PRR greater than 120 g subgroup. On the basis of the fixed effect model $(P>0.10)$, the ORs with $95 \%$ CI of the three subgroups were [OR: 3.46, 95\% CI $(2.23,5.37)$ ] (PRR in 30-60 g), [OR: 3.76, 95\% CI $(2.12,6.69)$ ] (PRR in 60$120 \mathrm{~g}$ ), and [OR: 6.15, 95\% CI $(3.03,12.47)$ ] (PRR more than 120 $\mathrm{g})$, respectively. All three of the subgroups showed a significant increase in the efficacy rate of the trial group compared with the control group $(P<0.01)$. Furthermore, the efficacy was enhanced with an increasing dosage of PRR in relevant formulae (Figure 9).

\section{Adverse Events}

Among the 15 RCTs, two articles (Li and Gu, 1994; Bo, 2008) reported no side effects in clinical trials. Another three studies (Xu et al., 1999; Wang et al., 2003; Lou et al., 2007) observed side effects as light digestive tract symptoms in patients treated with formulae of high-dose PRR. However, the side effect did not influence the patients' efficacy or daily life. The remaining articles did not report whether there was a side effect. Despite the results suggesting that high-dose PRR is safe, more assessments on the safety of high-dose PRR are needed (Table 2).

\section{Publication Bias}

A funnel plot was used to express the publication bias. The publication bias was explored when the indices or efficacy rate were above 9 cases. A funnel plot of the formulae of high-dose PRR combined with essential treatment vs. essential treatment was applied. Further, the indices of TBIL and ALT were also analyzed. The plots were symmetric, suggesting that there was no obvious publication bias (Figure 10).

\section{DISCUSSION}

As we mentioned, cholestatic hepatitis, and hyperbilirubinemia are both related to the dysfunction of bilirubin metabolism. However, there are several differences between cholestatic hepatitis. Cholestatic hepatitis is more concerned about the whole progression of the disease which could result in partial or complete blocking bile flow, high serum concentrations of bile acid, bilirubin, and liver injury. On the other side, hyperbilirubinemia could be widely expressed during the specific progression of various liver diseases and is more focused on the manifestation of high bilirubin. Moreover, the degree of bilirubin in hyperbilirubinemia is usually far more severe than in cholestatic hepatitis. In our former research, we have investigated the effect of high dose PRR relevant formulae on cholestatic hepatitis and found that this therapy could promote the efficacy (Ma et al., 2014). Therefore, according to the former result, we performed this meta-analysis on hyperbilirubinemia triggered by viral hepatitis. This systematic review included 15 RCTs; a total of 1323 participants with hyperbilirubinemia were screened from 4300 publications. All of the studies were based on highdose PRR (30-200 g) serving as the primary component of the formulae. The treatment duration described by the RCTs ranged from 2 weeks to 60 days, and five of the 15 studies reported side effects. According to the outcome, a single application or a combination of high-dose PRR formulae promoted efficacious hyperbilirubinemia treatment. The indices of hyperbilirubinemia also verified the result. TBIL is considered a direct index of hyperbilirubinemia. The serum TBIL level in 12 trials was analyzed by the random effects model. The MD with the 95\% CI of the serum TBIL level was [MD: -75.57 , 95\% CI $(-94.88,-56.26)]$ and indicated that formulae of high-dose PRR significantly decreased the serum TBIL level compared with 


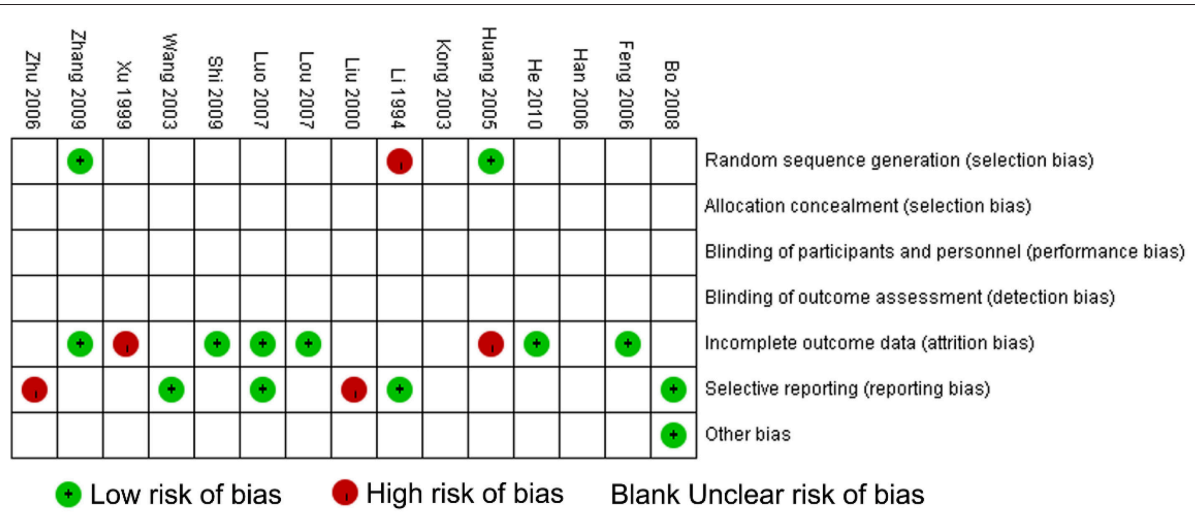

FIGURE 3 | Methodological quality assessment for risk of bias for each included study. Risk of bias was used to assess the quality of RCTs, and the majority of studies were found to be of low quality.

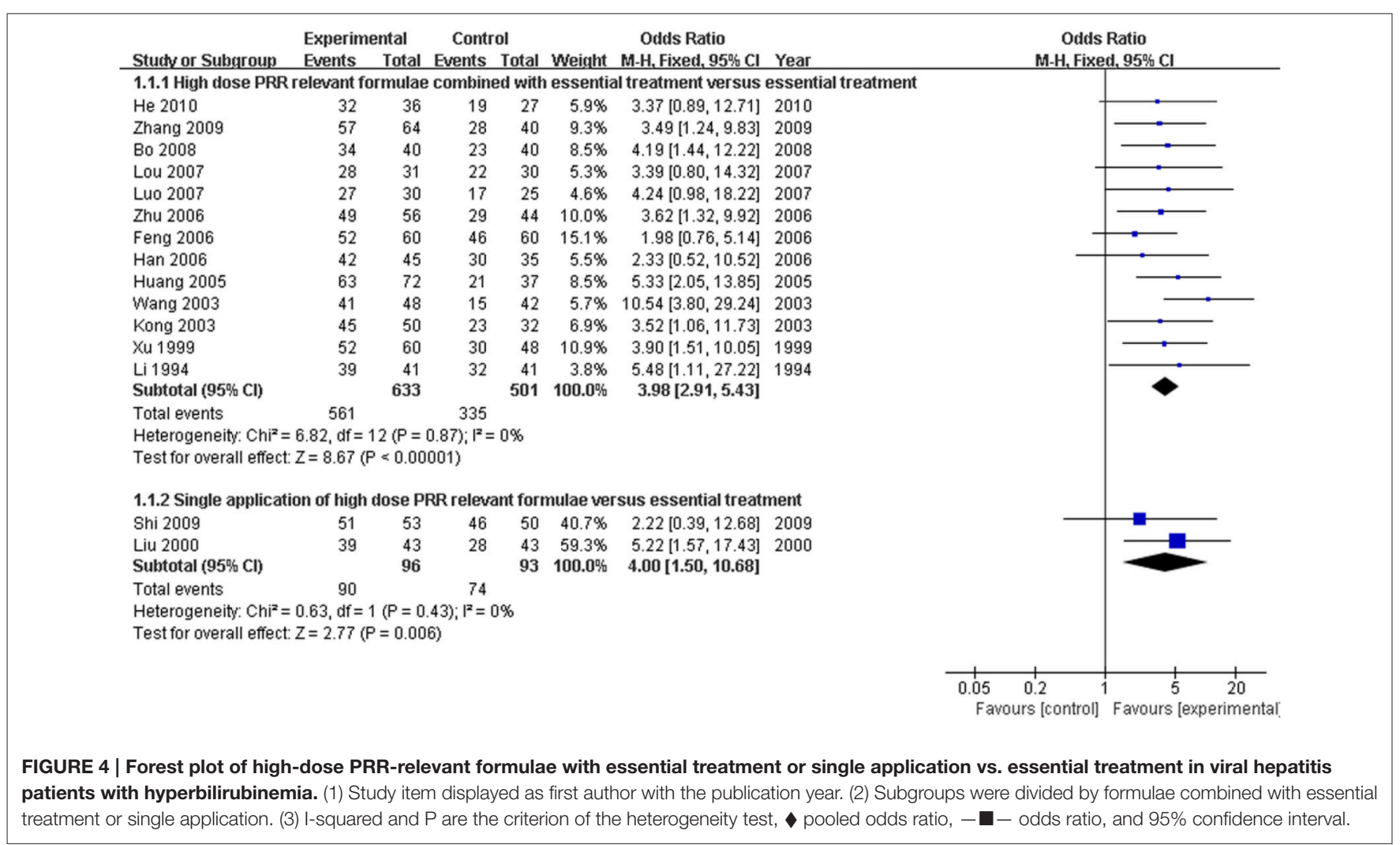

essential treatment. Furthermore, the serum ALT and AST levels, which are sensitive indices of hepatic function, also decreased. However, there were no differences in DBIL, $\gamma$-GT, PT, and ALB between the groups. This suggested that formulae of highdose PRR might promote the regulation of the main indices of hyperbilirubinemia. Hepatitis B virus is the most common and important cause of hyperbilirubinemia. Therefore, the efficacy of high-dose PRR formulae on hyperbilirubinemia triggered by hepatitis B was specifically analyzed in this study. The result showed a significant increase in the efficacy rate in the trial group compared with the control group. This suggested that formulae of high-dose PRR might promote efficacious treatment of the hyperbilirubinemia caused by hepatitis B. Because there was a wide range of high-dose PRR, from 30 to $200 \mathrm{~g}$, we performed an analysis on the efficacy rate of different ranges of high-dose PRR formulae. All three subgroups showed a significant increase in the efficacy rate of the trial group. Furthermore, the efficacy enhanced as the dosage of PRR in the relevant formulae was increasing. There were a few patients with light side effects of the digestive tract in the trial group. However, it did not influence the treatment progress and efficacy. A funnel plot was applied to assess the publication bias. The result indicated that little 


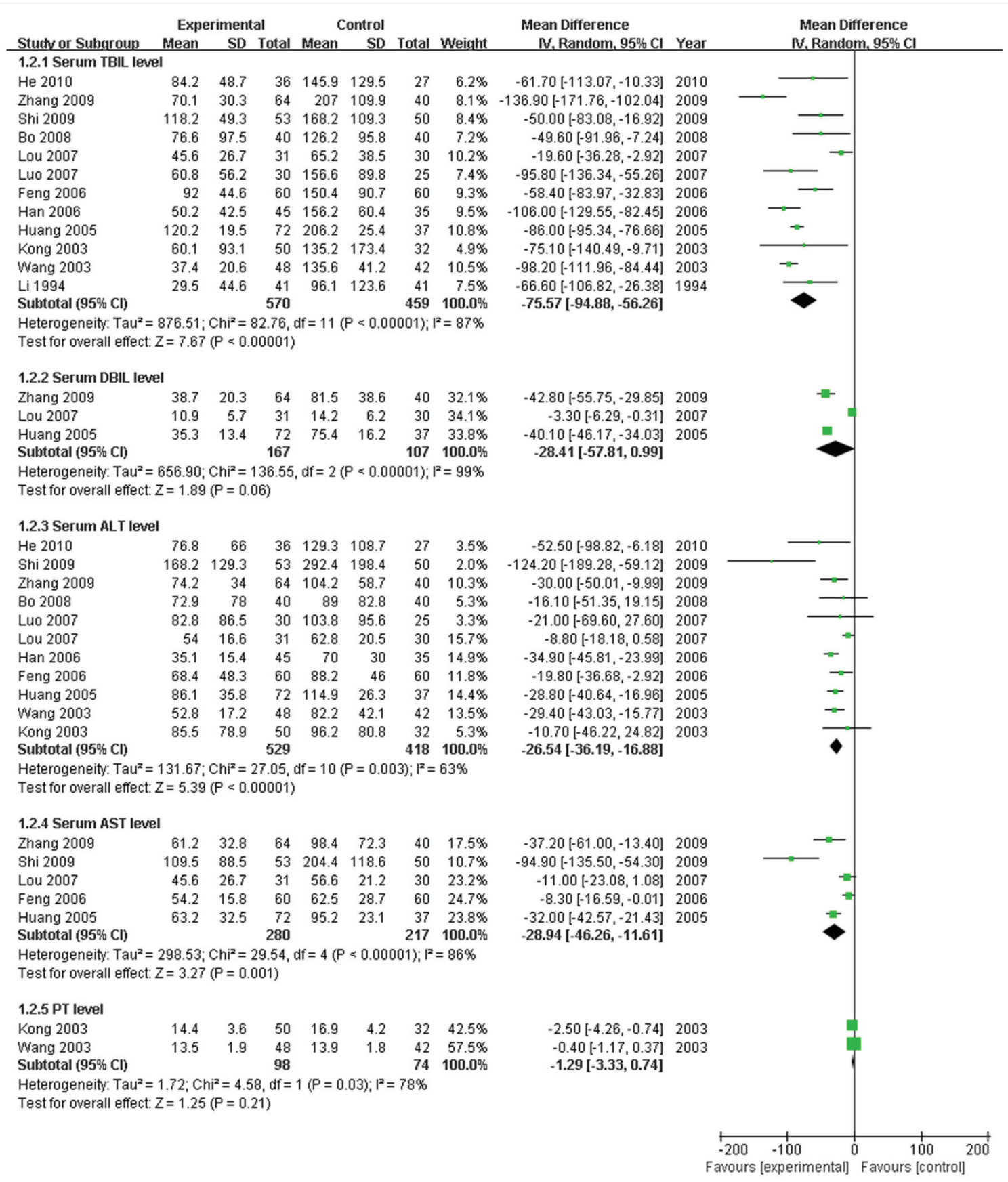

FIGURE 5 | Forest plot of TBIL, DBIL, ALT, AST, and PT in treatment. (1) Study item displayed as first author with the publication year. (2) Subgroups were divided by indices of TBIL, DBIL, ALT, AST, and PT. (3) I-squared and P are the criterion of the heterogeneity test, $\downarrow$ pooled mean difference, $-\mathbf{\square}-$ mean difference, and $95 \%$ confidence interval.

publication bias occurred in terms of efficacy and indices. This systematic review illustrates that formulae of high-dose PRR are safe to promote the efficacious treatment of hyperbilirubinemia.

Hyperbilirubinemia, presenting with severe jaundice, is one of the most common manifestations in viral hepatitis patients. It usually leads to severe necrosis of hepatocytes over a chronic period (Wang, 2010). It is well-known that a variety of definite factors, including infection, drug abuse, autoimmunity, and heredity, are able to cause hyperbilirubinemia. As for the mechanism, researchers have mainly focused on disorders of bilirubin metabolism such as uptake, synthesis, and excretion (Nie, 2006). In detail, repeated inflammation in viral hepatitis always acts as the trigger of jaundice and ultimately results in hepatocyte damage and even necrosis. Once hepatocytes are 


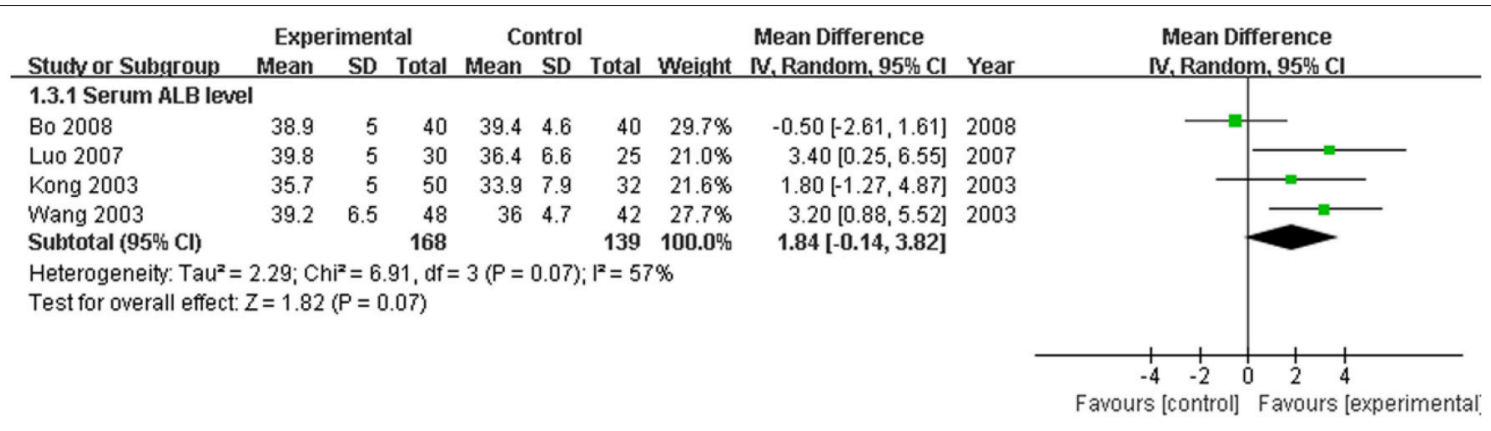

FIGURE 6 | Forest plot of ALB in treatment. (1) Study item displayed as the first author with the publication year. (2) The index of serum ALB was assessed in RCTs. (3) I-squared and P are the criterion of the heterogeneity test, $\downarrow$ pooled mean difference, $-\mathbf{\square}-$ mean difference, and $95 \%$ confidence interval.

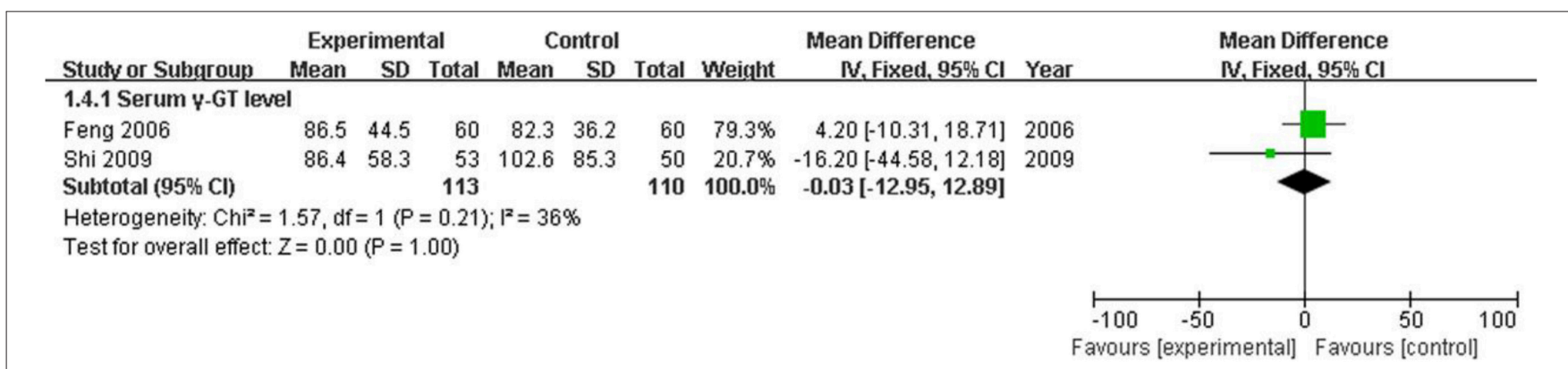

FIGURE 7 | Forest plot of $\gamma$-GT in treatment. (1) Study item displayed as the first author with the publication year. (2) The index of serum $\gamma$-GT was assessed in RCTs. (3) I-squared and P are the criterion of the heterogeneity test, $\downarrow$ pooled mean difference, $-\boldsymbol{-}-$ mean difference, and 95\% confidence interval.

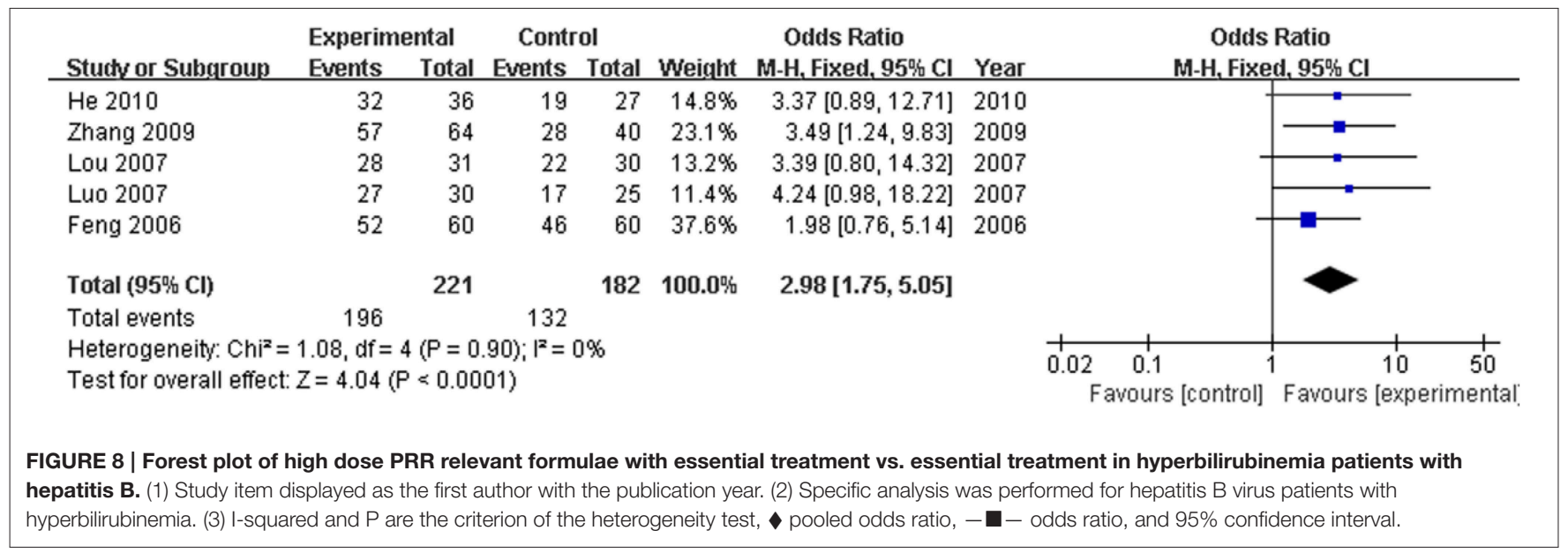

damaged, the metabolism of bilirubin is disturbed, thus causing bilirubin accumulation. If the high concentration of bilirubin fails to be eliminated, it accumulates such that hyperbilirubinemia or jaundice occurs. Moreover, a micro-imbalance would also occur in hepatocytes, such as inhibition of $\mathrm{Na}^{+}-\mathrm{K}^{+}$-ATPase, down regulation of NTCP and OATP expression, and deregulation of CAR and PXR expression. All of these consequences lead to bilirubin deposition in hepatocytes and jaundice and can cause worsening of hepatocyte damage (Paumgartner and Beuers, 2002; Wagner et al., 2011; Wlcek et al., 2013). According to the mechanism, hyperbilirubinemia is associated with various tissues and targets. Current western medical treatment for hyperbilirubinemia mainly focuses on the combination of alleviating jaundice, liver protection, lowering transaminase levels, nutrition support, etc. However, it lacks comprehensive adjustment and has unstable efficacy. In contrast, complementary and alternative medicine such as TCM for the treatment of hyperbilirubinemia often obtains positive results.

$P R R$ has been widely used in TCM practice for thousands of years for the treatment of blood stasis by targeting the liver. Current studies indicate that PRR and its active components consistently exhibit wide pharmacological effects 


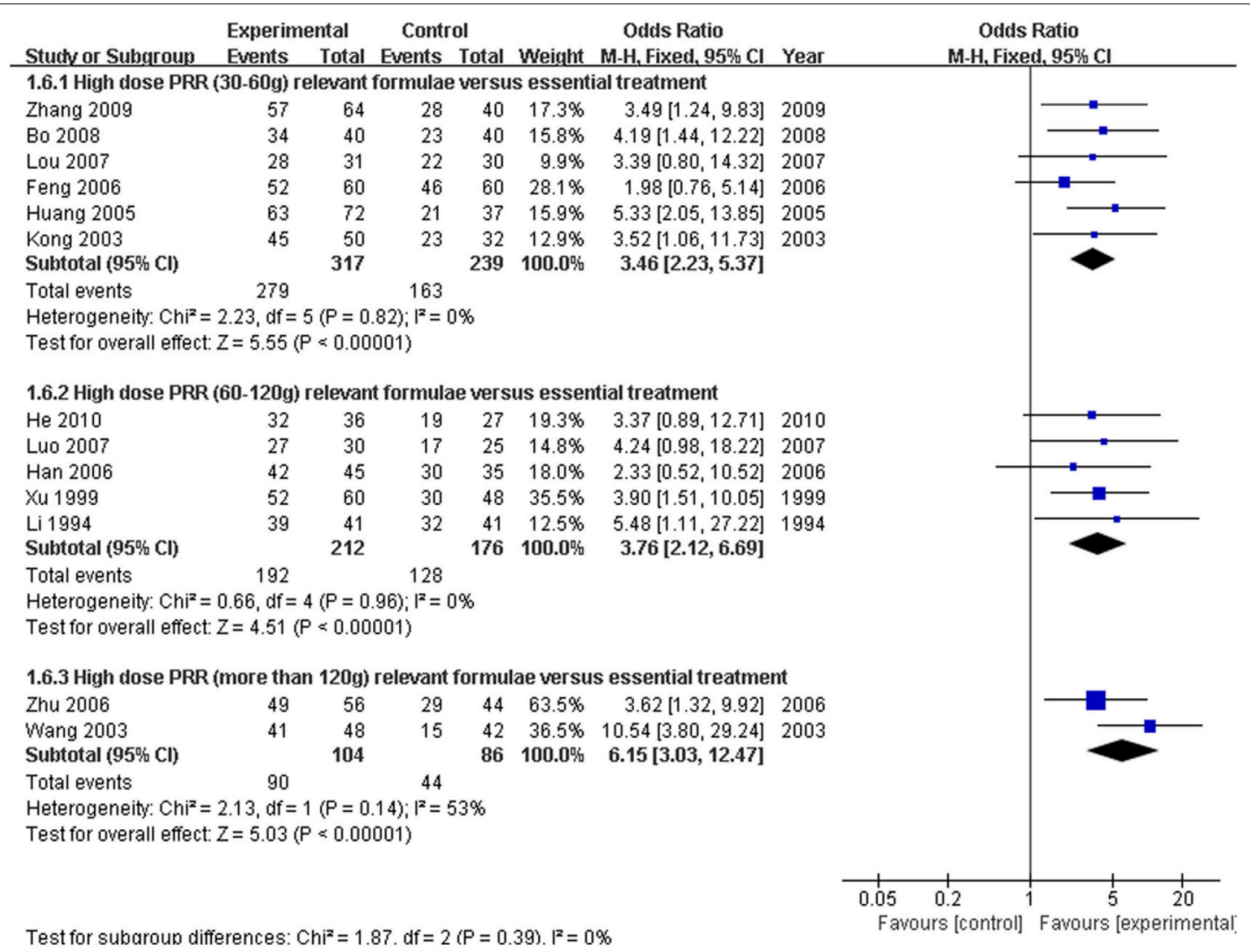

FIGURE 9 | Forest plot of the different ranges of high-dose PRR-relevant formulae combined with essential treatment vs. essential treatment alone. (1) Study item displayed as the first author with the publication year. (2) Subgroups were classified as PRR 30-60 g, PRR 60-120 g and PRR greater than 120 g. (3) $\mathrm{I}$-squared and $\mathrm{P}$ are the criterion of the heterogeneity test, $\bullet$ pooled odds ratio, $-\mathbf{-}-$ odds ratio, and $95 \%$ confidence interval.

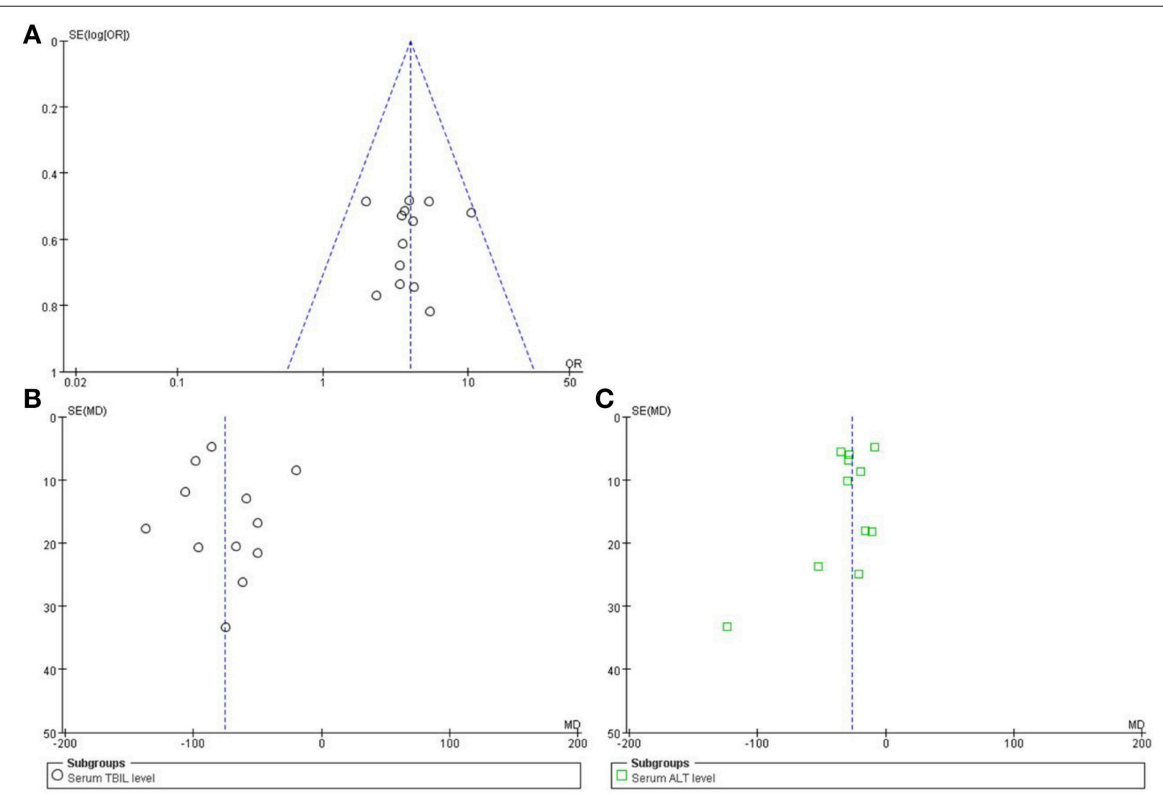

FIGURE 10 | Funnel plot of high-dose PRR-relevant formulae. (A) The plot of efficacy rate, (B) serum index of TBIL, and (C) serum index of ALT was symmetric, suggesting that the publication bias was small. 
such as vasodilatation of the thoracic aorta (Jin et al., 2010), liver protection (Zhao et al., 2013), an anti-allergic effect (Lee et al., 2008), anti-inflammation, and immunoregulation ( $\mathrm{He}$ and Dai, 2011). According to the TCM theory and modern pharmacology, the original clinical practice of formulae of high-dose PRR to treat hyperbilirubinemia has been gradually applied in recent years (Zhu, 2011). In addition, some studies may partially reveal the mechanism of this application. PRR is able to promote bile acid excretion, enhance the activity of UDPGT and decrease the $\mathrm{TXB}_{2}$ level (Wang, 1986; Lei et al., 1988). In this aspect, PRR is effective in decreasing bilirubin. Although, there is efficacy on hyperbilirubinemia, it remains unclear why PRR should be used at such a high dose. Clinical studies have shown that there is a significant difference in efficacy between the high-dose treatment group $(30 \mathrm{~g})$ and the normal-dose treatment group $(10 \mathrm{~g})$. The efficacy of the highdose treatment group is markedly higher than the normal-dose treatment group in alleviating hyperbilirubinemia $(P<0.01)$ (Luo, 2007). This indicates that low-dose PRR cannot effectively treat hyperbilirubinemia compared with a high dose. This is likely related to the characteristic active compounds in PRR. Our previous study demonstrated that the bioavailability of paeoniflorin and albiflorin in PRR is extremely low (Jiang et al., 2012). Herein, a high dose of PRR shows stable treatment efficacy for the alleviation of hyperbilirubinemia, which was not found at low doses. According to these results, formulae of high-dose PRR might help uncover the crucial and specific function of this therapy for hyperbilirubinemia.

Currently, increasing attention has focused on TCM. Due to the holistic concept and treatment based on syndrome

\section{REFERENCES}

Bo, T. (2008). Clinical Study of Jia Wei Wen Dan Decoction on Treating Hyperbilirubinemia with Viral Hepatitis. Wuhan: Hubei University of Chinese Medicine.

Ding, Q. X., Liu, T. D., and Wang, L. X. (2003). Analysis on compound Chishao Decoction in treating refractory hyperbilirubinemia. Chin. J. Dis. Control. Prev. 7, 478. doi: 10.3969/j.issn.1674-3679.2003.05.045

Duan, Z. J., Li, L. L., Ju, J., Gao, Z. H., and He, G. H. (2006). Treatment of hyperbilirubinemia with blood purification in China. World. J. Gastroenterol. 12, 7467-7471. doi: 10.3748/wjg.v12.i46.7467

Feng, H. P., Guo, X. P., Zhong, D. J., Zhang, J. H., Cao, Q. J., and Liu, Y. W. (2006). Clinical observation of Da Huang Chi Shao Decoction on treating hyperbilirubinemia induced by viral hepatitis. J. Mod. Med. Health 22, 64. doi: 10.3969/j.issn.1009-5519.2006.01.061

Garg, H., Sarin, S. K., Kumar, M., Garg, V., Sharma, B. C., and Kumar, A. (2011). Tenofovir improves the outcome in patients with spontaneous reactivation of hepatitis B presenting as acute-on-chronic liver failure. Hepatology 53, 774-780. doi: 10.1002/hep. 24109

Han, Z. W., Liu, J. M., and Han, P. X. (2006). Analysis on integrative medical treatment for hyperbilirubinemia with viral hepatitis. Mod. J. Integr. Tradit. Chin. West. Med. 15, 1764-1765. doi: 10.3969/j.issn.1008-8849.2006.13.031

He, D. Y., and Dai, S. M. (2011). Anti-inflammatory and immunomodulatory effects of paeonia lactiflora pall, a traditional chinese herbal medicine. Front. Pharmacol. 2:10. doi: 10.3389/fphar.2011.00010

He, J. P., Wang, C. B., Ding, J. B., Liu, F. Q., Han, J., Zhao, Y. L., et al. (2003). Open comparative study of Chidan Tuihuang Granule in treating acute and chronice viral cholestatic hepatitis. C.J.I.T.W.M 23, 498-500. doi: 10.3321/j.issn:10035370.2003.07.006 differentiation, TCM emphasizes formulae employment instead of a single drug application in treating diseases. Many studies demonstrate that formulae with favorable efficacy for specific diseases regulate body function in various organs and tissues in a network manner (Li et al., 2007; Li and Zhang, 2013). Therefore, further studies to illustrate the potential mechanism should be explored.

\section{CONCLUSION}

Formulae of high-dose PRR might safely promote the efficacious treatment of hyperbilirubinemia and are thus a promising treatment approach. For acceptance by widespread practitioners, more rigorously designed multicenter, doubleblind, randomized, and large-scale controlled trials are required for further investigation.

\section{AUTHOR CONTRIBUTIONS}

$\mathrm{YH}, \mathrm{XM}$, and JW analyzed the data and wrote the manuscript. YZ, ZC, and WZ collected and prepared samples. JW, JW, LS performed the analyses. YZ and XX designed the study and amended the paper.

\section{ACKNOWLEDGMENTS}

The current work was supported by the Major Projects of the National Science and Technology (no.2012ZX10005010-002002) and the National Natural Science Foundation Project of China (no.81303120) and (no.81173571).

He, Q. Z. (2010). Observation on combination of Tui Huang Decoction and western medicine in treating 36 cases of hyperbilirubinemia. Hebei. J. TCM 32, 714-715. doi: 10.3969/j.issn.1002-2619.2010. 05.043

Hou, G. H., Hu, Q. J., Wang, H. L., Li, M., Xu, M. H., Zhu, S. Y., et al. (2010). Adjuvant enema medicine treatment of 54 cases viral hepatitis hyperbilirubinemia. Chin. J. Integr. Trad. West. Med. Dig. 18, 398-400. doi: 10.3969/j.issn.1671-038X.2010.06.015

Huang, J. S., and Yang, R. Q. (2005). Clinical observation on treating hyperbilirubinemia in viral hepatitis with Liang Xue Hua Yu Decoction. J. Sichuan Tradit. Chin. Med. 23, 58-59. doi: 10.3969/j.issn.10003649.2005.03.033

Jiang, F. J., Zhao, Y. L., Wang, J. B., Wei, S. S., Wei, Z. M., Li, R. S., et al. (2012). Comparative pharmacokinetic study of paeoniflorin and albiflorin after oral administration of Radix Paeoniae Rubra in normal rats and the acute cholestasis hepatitis rats. Fitoterapia 83, 415-421. doi: 10.1016/j.fitote.2011. 12.009

Jin, S. N., Wen, J. F., Wang, T. T., Kang, D. G., Lee, H. S., and Cho, K. W. (2010). Vasodilatory effects of ethanol extract of Radix Paeoniae Rubra and its mechanism of action in the rat aorta. J. Ethnopharmacol. 142, 188-193. doi: 10.1016/j.jep.2012.04.035

Kong, Q. H., Lan, Y., Li, B., and Zhou, A. P. (2003). Combination of Yin Chen Chi Ling Decoction and western medicine in treating 50 cases of severe jaundice in viral hepatitis. Chin. J. Integr. Tradit. West. Med. Liver Dis. 13, 234-235. doi: 10.3969/j.issn.1005-0264.2003.04.023

Lee, B., Shin, Y. W., Bae, E. A., Han, S. J., Kim, J. S., Kang, S. S., et al. (2008). Antiallergic effect of the root of Paeonia lactiflora and its constituents paeoniflorin and paeonol. Arch. Pharm. Res. 31, 445-450. doi: 10.1007/s12272001-1177-6 
Lei, Z. Y., Xu, J. H., Sun, J. F., Li, X. L., Du, X. Y., Tian, N., et al. (1988). Study on hepatic microcirculation of intrahepatic cholestasis. Med. J. Chin. Peoples Liberation Army 13, 382-384

Li, S. B., and Gu, R. W. (1994). Observation on treating hyperbilirubinemia in viral hepatitis with high dosage of Chishao. Zhejiang J. Integr. Tradit. Chin. West. Med. 4, 16-17.

Li, S., and Zhang, B. (2013). Traditional Chinese medicine network pharmacology: theory, methodology and application. Chin. J. Nat. Med. 11, 110-120. doi: 10.3724/SP.J.1009.2013.00110

Li, S., Zhang, Z. Q., and Wu, L. J. (2007). Understanding ZHENG in traditional Chinese medicine in the context of neuro-endocrine-immune network. IET Syst. Biol. 1, 51-60. doi: 10.1049/iet-syb:20060032

Li, Y. M., Yang, H. Z., Guan, W. B., Ke, Q. S., Dai, M., Xie, H. P., et al. (2008). Therapeutic effect of traditional Chinese medicine on coagulation disorder and accompanying intractable jaundice in hepatitis B virus-related liver cirrhosis patients. World J. Gastroenterol. 14, 6060-6064. doi: 10.3748/wjg. 14.6060

Liu, Y. (2000). Treatment on 86 cases of severe jaundice hepatitis with high dose Radix Paeoniae Rubra. J. New Chin. Med. 12:40. doi: 10.3969/j.issn.02567415.2000.12.022

Lou, H. M., Li, Z. G., and Wang, Y. Y. (2007). Clinical analysis of treating hyperbilirubinemia in viral hepatitis with Tui Huang Decoction. Mod. J. Integr. Tradit. Chin. West. Med. 16, 4939-4940. doi: 10.3969/j.issn.10088849.2007.33.021

Luo, X. B. (2007). Clinical observation on detoxification and promoting blood flow decoction using large dosage Paeoniae Radix for the treatment of type B hepatitis hyperbilirubinemia. Guid. J. TCM 13, 16-17.

Ma, X., Wang, J., He, X., Zhao, Y. L., Wang, J. B., Zhang, P., et al. (2014). Large dosage of chishao in formulae for cholestatic hepatitis: a Systematic review and meta-analysis. Evid. Based Complement. Alternat. Med. 2014:328152. doi: $10.1155 / 2014 / 328152$

Nie, Q. H. (2006). Relevant disease of bilirubin metabolic disturbance. Chin. Hepatol. 11, 189-192. doi: 10.3969/j.issn.1008-1704.2006.03.016

Paumgartner, G., and Beuers, U. (2002). Ursodeoxycholic acid in cholestatic liver disease: mechanisms of action and therapeutic use revisited. Hepatology 36, 525-531. doi: 10.1053/jhep.2002.36088

Shi, J., Qiang, J., Tan, G. M., Wang, P. J., and Li, L. Q. (2009). Clinical observation of clearing heat, cooling blood and detoxicating principle on treating chronic severe jaundice with viral hepatitis B. J. E. T. C. $M$ 18, 884-885. doi: 10.3969/j.issn.1004-745X.2009.06.019

Wagner, M., Zollner, G., and Trauner, M. (2011). Nuclear receptors in liver disease. Hepatology 53, 1023-1034. doi: 10.1002/hep.24148

Wang, C. B. (1986). Effect and mechanism of cooling blood and invigorating blood circulation herb in treating severe jaundice hepatitis. Chin. J. Integr. Med. 27, 271-272.
Wang, C. B. (2010). Integrative Medical Treatment in Liver Disease with Severe Jaundice. Beijing: People's Military Medical Press.

Wang, L. J., Zhu, Y. G., Li, M., Zhang, Y. Y., and Dou, Z. H. (2003). Clinical observation on treating 120 cases of severe jaundice with Tui Gao Huang Decoction. Jiangsu J. Tradit. Chin. Med. 24, 15-17. doi: 10.3969/j.issn.1672397X.2003.09.007

Wlcek, K., Koller, F., and Ferenci, P. (2013). Hepatocellular organic aniontransporting polypeptides (OATPs) and multidrug resistance-associated protein 2 (MRP2) are inhibited by silibinin. Drug Metab. Dispos. 41, 1522-1528. doi: $10.1124 / \mathrm{dmd} .113 .051037$

Xu, H. J., Su, T. B., and Wang, Y. Z. (1999). Chi Zhi Huang Decoction in treating 60 cases of severe jaundice with viral hepatitis. J. Henan Univ. Chin. Med. 14, 32-33.

Zhang, L. L. (2009). Integrative medical treatment for 64 cases of chronic hyperbilirubinemia induced by viral hepatitis B. Hunan J. Tradit. Chin. Med. 25, 58-59. doi: 10.3969/j.issn.1003-7705.2009.05.035

Zhang, M. F., and Shen, Y. Q. (2011). Re-evaluation of therapeutic effect of glycyrrhizic acid on hepatopathy. Anti. Infect. Pharm. 8, 82-87. doi: 10.3969/j.issn.1672-7878.2011.02-002

Zhao, Y. L., Zhou, G. D., Wang, J. B., Jia, L., Zhang, P., and Li, R. S. (2013). Paeoniflorin protects against ANIT-induced cholestasis by ameliorating oxidative stress in rats. Food Chem. Toxicol. 58, 242-248. doi 10.1016/j.fct.2013.04.030

Zhou, G. L. (2010). Research on Ancient Formulae Resource and Drug Application Principle in Treating Jaundice. Shenyang: Liaoning University of Chinese Medicine.

Zhu, G. H., Lan, N., and Wang, Y. Z. (2006). High dose Paeoniae Radix Rubra in treating 56 cases of acute hyperbilirubinemia with viral hepatitis. J. Mod. Med. Health 22, 1717. doi: 10.3969/j.issn.1009-5519.2006.11.114

Zhu, Y. (2011). Professor Wang Chengbo's experience of high dosage of promoting Qi flow and blood circulation herbs in treating liver disease. Chin. J. Integr. Tradit. West. Med. Liver Dis. 21, 105-108. doi: 10.3969/j.issn.10050264.2011.02.018

Conflict of Interest Statement: The authors declare that the research was conducted in the absence of any commercial or financial relationships that could be construed as a potential conflict of interest.

Copyright (C) 2016 Huang, Ma, Wang, Zhao, Wang, Chen, Zhu, Shan, Wei, Wang and Xiao. This is an open-access article distributed under the terms of the Creative Commons Attribution License (CC BY). The use, distribution or reproduction in other forums is permitted, provided the original author(s) or licensor are credited and that the original publication in this journal is cited, in accordance with accepted academic practice. No use, distribution or reproduction is permitted which does not comply with these terms. 